Int. J. Dev. Biol. 49: 491-500 (2005)

doi: $10.1387 / \mathrm{ijdb} .041968 \mathrm{pm}$

\title{
Circadian clock signaling in Arabidopsis thaliana: from gene expression to physiology and development
}

\author{
PALOMA MÁS* \\ Consorcio CSIC-IRTA, Laboratorio de Genética Molecular Vegetal, Instituto de Biología Molecular de Barcelona (IBMB-CSIC), \\ Barcelona, Spain
}

\begin{abstract}
The daily rotation of the earth on its axis leads to predictable periodic fluctuations of environmental conditions. Accordingly, most organisms have evolved an internal timing mechanism, the circadian clock, which is able to recognize these 24-hour rhythmic oscillations. In plants, the temporal synchronization of physiology with the environment is essential for successful plant growth and development. The intimate connection between light signaling pathways and the circadian oscillator allows the anticipation of the environmental transitions and the measurement of day-length as an indicator of changing seasons. In recent years, significant advances have been made in the genetic and molecular dissection of the plant circadian system, mostly in Arabidopsis thaliana. The overall plant clock organization is highly complex; the system seems to include several input pathways, tightly regulated central oscillators and a myriad of outputs. The molecular cloning and characterization of a number of clock components has greatly improved our view of the plant central oscillator and additional players will most likely come into place very soon. Molecular mechanisms underlying circadian clock function are also beginning to be characterized. The emerging model relies on negative feedback loops at the core of the oscillator. Additional levels of post-transcriptional and post-translational regulation also contribute to the generation and maintenance of the rhythms. Globally, these studies have shed new light on how the clock coordinates plant physiology and development with the daily and seasonal environmental cycles.
\end{abstract}

KEY WORDS: biological clock, circadian rhythms, Arabidopsis thaliana

The biological clock entails a 24-hour rhythm on biochemical and physiological processes such that they occur at specific, advantageous times of the day-night cycle (Dunlap, 1999, Young and Kay, 2001). The circadian movements of leaves and flowers, the fragrance emission, the stomatal opening, hypocotyl expansion or the photoperiodic control of flowering time are some examples of processes tightly regulated by the plant clockwork (Barak et al., 2000, McClung, 2001). Underlying all these physiological rhythms are endogenous circadian oscillations of gene expression. Indeed, genomic approaches have identified in Arabidopsis hundreds of genes under clock control, with peaks of expression at all phases of the day/night cycle (Harmer et al., 2000, Schaffer et al., 2001). Although the rhythmic oscillations persist in constant conditions (i.e. in the absence of environmental transitions) the circadian clock does not run in isolation from the environment. The clock includes a resetting mechanism by which it is synchronized each day to the correct time (Devlin and Kay, 2001). The presence of an endogenous timing system provides an adaptive advantage, enabling the anticipation of the environmental transi- tions and the temporal coordination of physiological events to occur at specific phase relationships with the environment (Johnson, 2001). Thus, the circadian clock can be considered as an internal processor of environmental signals (such as light and temperature) that coordinates the appropriate timing of metabolic and developmental activities in the plant (Harmer et al., 2001, McClung, 2001).

Classically, the circadian system has been divided into three main components: the inputpathways involved in the perception and transmission of environmental signals to synchronize the central oscillator or pacemaker that generates and maintains rhythmicity through multiple output pathways that connect the oscillator to physiology and metabolism (Figure 1). Clearly, this is an oversimplified conceptual model of the clock. Several lines of evidence reveal the existence of a far more complicated circadian system, with output elements modulating the pace of the oscillator and input elements being themselves tightly controlled by the clock. The existence of different free-running periods in the expression of diverse clock outputs is indicative of separate

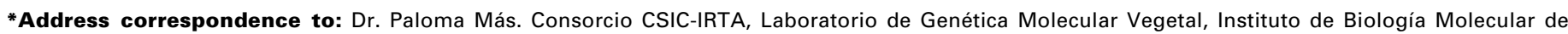
Barcelona (IBMB-CSIC), C/ Jordi Girona 18-26, 08034 Barcelona, Spain. Fax: +34-94-204-5904. e-mail: pmmgmc@ibmb.csic.es
} 


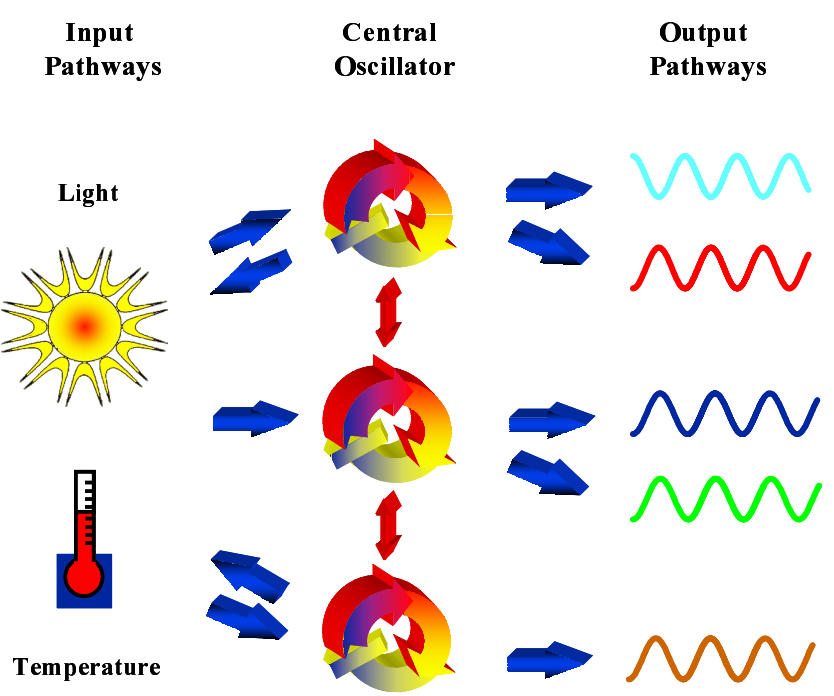

Fig. 1. Organization of the circadian clock in plants. Input pathways, such as light and temperature, connect the clock with the external environment. Multiple central oscillators are depicted as feedback loops with possible coupling among them. Positive and negative elements in the loops yield self-sustained oscillation. Output from the oscillators produces rhythms that can differ in phase. Some outputs might be driven by individual oscillators whereas others might receive input from more than one.

oscillators that might share common components and mechanisms in distinct locations (Eriksson and Millar, 2003, Hall et al., 2002, Michael et al., 2003a, Thain et al., 2002). In this review, I summarize some of the highlights discovered on the plant circadian world: the cloning and identification of clock components and the few described mechanisms and signaling pathways in the Arabidopsis clockwork. This review pretends to portray a general view of how the circadian clock permeates important aspects of plant physiology and development. A single review can not do justice to all the relevant studies in the field and therefore, I apologize for those whose work does not appear herein. Excellent reviews cover in more detail specific aspects of the circadian research and the readers are encouraged to consult them.

\section{The core players in the Arabidopsis circadian sys- tem}

The identification of molecular components in the circadian system was facilitated by the use of plants expressing the promoter of the clock-controlled gene $\angle H C B$ or $C A B$ (lightharvesting chlorophyll $\mathrm{a} / \mathrm{b}$ protein) fused to the firefly $\angle U$ CIFERASE (LUC). The CAB promoter drives a robust rhythm of bioluminescence that can be monitored with a photoncounting video camera (Millar et al., 1995). Mutagenesis of these transgenic plants followed by bioluminescence analysis of abnormal $C A B$ rhythms resulted in the isolation of a number of circadian period mutants. One of these mutants, the timing of $C A B$ expression 1 (toc 1-1) displayed a shorter than wild-type circadian period not only for $C A B:: L U C$ expression but also for other circadian outputs under a wide range of temperature and light conditions (Millar et al., 1995). The toc1-1 mutant plants also exhibited a day-length-insensitive, early-flowering phenotype, under cycles of $24 \mathrm{~h}$, but not under $21 \mathrm{~h}$ environmental cycles (which match the endogenous period of this mutant (Strayer et al., 2000). This indicates that the photoperiodic insensitivity is due to the improper functioning of the clock caused by the toc1-1 mutation. Cloning of the gene revealed that TOC1 encoded a nuclear protein containing a receiver domain similar to the one found in plant response regulators (Strayer et al., 2000). However, the conserved phospho-accepting aspartate residues present in bona fide response regulators is missing in TOC1 (also denominated pseudoresponse regulator), suggesting that it does not function in a canonical phosphor-relay mechanism (Mizuno, 2004). In addition, TOC1 contains a distinctive $\mathrm{COOH}$-terminal motif (CCT motif) which is conserved within the CONSTANS (CO) family of plant transcription factors (Strayer et al., 2000). Analysis of TOC1 expression revealed that the mRNA rhythmically cycled and participated in a negative feedback loop mechanism to control its own expression (Strayer et al., 2000). Together, these observations led to the idea that TOC1 was an important component of the core of the oscillator, rather than part of the light input pathway to the clock. However, a detailed characterization of TOC 1 function using RNAi plants and a strong allele of TOC1 (toc1-2) provided evidence of unpredicted roles for TOC1 in the control of circadian and photo-morphogenic responses (Más et al., 2003a). These studies showed that silencing of the TOC1 gene caused arrhythmia in constant darkness and in various intensities of red light. In addition, TOC1 RNAi and toc1-2 mutant plants displayed an important reduction in sensitivity to red and far-red light in the control of hypocotyl elongation whereas increments in TOC1 gene dosage clearly enhanced light sensitivity (Más et al., 2003a). Thus, based on these studies, it was postulated a new role for TOC1 in the integration of light signals from phytochromes to clock outputs, controlling circadian gene expression and other light-dependent developmental processes in the plant.

Two transcription factors, CCA1 (CIRCADIAN CLOCK ASSOCIATED 1) and LHY (LATE ELONGATED HYPOCOTYL), with a single MYB-like domain, have also been closely associated with the plant circadian system (Schaffer et al., 1998, Wang and Tobin, 1998). CCA1 was identified as a binding factor to a region of the promoter of Arabidopsis $\angle H C B$ gene. These initial studies indicated that CCA1 protein was an important element in the functioning of the phytochrome signal transduction leading to increased transcription of the $\angle H C B$ gene (Wang et al., 1997). Later studies also revealed an additional role of CCA1 in the circadian clockwork. Loss of function cca 1 mutants displayed a shorter than wild-type period of genes expressed at different circadian times while CCA1 constitutive over-expression clearly disrupted rhythmicity in various clock outputs including hypocotyl elongation, leaf movements and circadian gene expression (Green and Tobin, 1999, Wang and Tobin, 1998).

The lhy mutation was caused by the insertion of a transposon (Ds) within the 5' untranslated region (5'UTR) of the $L H Y$ gene, causing its over-expression. Studies of these LHY overexpressing plants as well as loss of function /hy mutants showed clear alterations in circadian rhythmicity for leaf move- 
ment and for the circadian regulation of gene expression. Both $C C A 1$ and $\angle H Y$ transcripts rhythmically oscillate, with a peak of expression early in the morning, shortly after dawn. The rhythms persists in constant conditions (constant light and constant darkness) indicating that both genes are under circadian control (Schaffer et al., 1998, Wang and Tobin, 1998). The circadian expression of the $\angle H Y$ and $C C A 1$ transcripts is abolished in /hy1 and CCA1-overexpressing (CCA1-ox) plants, suggesting that the rhythmic expression of $\angle H Y$ and $C C A 1$ is required for normal circadian function. Interestingly, CCA1 and LHY regulated their own and each other's expression. Overexpressing plants of either gene exhibited arrhythmic expression of the endogenous $\angle H Y$ and CCA1 transcripts. Furthermore, the expression of both endogenous genes was repressed to levels similar to trough in wild-type plants. These results suggested that LHY and CCA1 might function redundantly as components of a negative feedback loop (Schaffer et al., 1998, Wang and Tobin, 1998). Indeed, recent analysis comparing phenotypes in single and double mutant Ihy-ccal plants indicated that the $\angle H Y$ and $C C A 1$ genes are partially redundant and that they are required for maintenance of circadian rhythmicity in Arabidopsis (Alabadí et al., 2002, Mizoguchi et al., 2002).

More recently, another player has joined the clockwork team, in very close association with the circadian oscillator. ELF4 (EARLY-FLOWERING 4) which encodes a protein of 111 amino acids without predictable protein domains, was identified by its involvement in photoperiodic perception and circadian regulation (Doyle et al., 2002). Analysis of plants containing a T-DNA insertion in the ELF4 gene revealed an early flowering phenotype in short photoperiods while under long-day conditions, the mutant plants flowered at about the same time as wild-type (Doyle et al., 2002). The elf4 mutation reduced CCA1 expression and affected the rhythms of clock-controlled genes expressed at different circadian times. Their expression rapidly became arrhythmic under constant conditions, but individual seedlings were transiently rhythmic with highly variable periods. These results suggest a role for ELF4 in maintaining the accuracy of the rhythms. Consistent with the early flowering phenotype in short days, the elf4 mutation increased expression of $C O$, a gene that has a relevant role in floral induction (see below).

\section{Synchronization with external time: light input to the clock}

The earliest experimental evidence of the existence of an internal time-keeping mechanism came with the observations that the daily leaf movement of Mimosa plants continued even when plants were placed in constant darkness. These observations demonstrated that the environmental changes were not required to trigger the rhythmic response and revealed one of the main properties characterizing circadian rhythmicity: its ability to persist in the absence of environmental cues. In constant conditions, the period of the rhythms is approximately, but not exactly 24-hours. To generate an accurate 24-hours period, the endogenous oscillator must be synchronized with the external, environmental time (Johnson, 2001). Environmental transitions between dawn and dusk help to adjust the endogenous period of the clock to exactly match the 24-hour period that we find in nature (Devlin and Kay, 2001). As light is an essential environmental signal in the synchronization of the clock with the outside world, a long-standing goal in the plant circadian field has been the identification of the components in the signal transduction pathway responsible for resetting the clock. Studies of photoreceptor-deficient mutants crossed into the $C A B:: L U C$ plants provided evidence that two classes of photoreceptors, phytochromes (PHY) and cryptochromes (CRY), participated in the light-driven entrainment of the Arabidopsis clock (Somers et al., 1998a). Four of the five phytochromes identified in Arabidopsis (PHYA, PHYB, PHYD and PHYE) act additively in the red-light input to the clock while Arabidopsis CRYPTOCHROME 1 (CRY1) acts as a clock photoreceptor for high and low fluences of blue light and both CRY1 and CRY2 act redundantly at intermediate fluences of blue light (Devlin and Kay, 2000, Somers et al., 1998a). Mutations in cry2 were also shown to affect entrainment in white light conditions (Más et al., 2000) suggesting an interaction of CRY2 with the phytochrome signaling pathway. Indeed, using Fluorescent Resonance Energy Transfer (FRET) microscopy, PHYB and CRY2 were shown to interact in vivo, co-localizing in nuclear speckles in response to light treatments (Más et al., 2000). Surprisingly, cryptochrome mutant plants also showed altered entrainment under red light suggesting that CRY1 is required for PHYA signaling to the clock in both red and blue light (Devlin and Kay, 2000). Quadruple mutants (lacking phy A, phyB, cry1 and cry2 ) maintained rhythmicity (Yanovsky et al., 2000), indicating that additional components participate in the light input pathway to reset the clock. Some possible candidates to perform this function are the gene family ZEITLUPE (ZTL), FLAVIN-BINDING, KELCH REPEAT, F-BOX 1 (FKF1) and LOV, KELCH $P R O T E I N 2$ (LKP2). The genes encode proteins that contain six kelch repeats as well as a LOV and an F-box domain. The LOV domain is highly similar to the one found in the blue light photoreceptors NPH1 (Arabidopsis) and WHITE COLLAR 1 (WC-1; Neurospora). The F-box is a motif found in proteins that act as adapters and bring specific substrates to ubiquitin protein ligase subunits for degradation (Craig and Tyers, 1999). The unique combination of these domains in the ZTL family of proteins suggests that they might mediate light-depended protein degradation of critical clock components. Indeed, a recent report has shown TOC1 as one of the ZTL substrates (see below and Más et al., 2003b). Analysis of $z t /$ mutant plants revealed a long period circadian phenotype that was dependent on light intensity (Somers et al., 2000) while over-expression of LKP2 resulted in arrhythmic expression of several clock-controlled genes (Schultz et al., 2001). The $f k f 1$ mutant was initially identified by its late flowering phenotype (Nelson et al., 2000) and more recently, its role in the photoperiodic control of $\mathrm{CO}$ expression has been described (Imaizumi et al., 2003). The possible function of these proteins in photo-entrainment might result from their direct interaction with photoreceptors. Indeed, ZTL has been shown to interact in vitro with PHYB and CRY1 (Jarillo et al., 2001) although it remains to be determined the biological relevance of these interactions in vivo.

The central oscillator can be also entrained by temperature (warm/cold) cycles. This feature has been used to analyze whether circadian mutants affect specifically light signaling 
input to the clock (Millar, 2004). A detailed phase response curve to temperature was recently published (Michael et al., 2003a) and new evidence suggest that the light- and temperature-sensing systems in plants might be connected, since photoreceptor signaling pathways can be temperature sensitive (Halliday and Whitelam, 2003, Mazzella et al., 2000). In spite of these new advances, we are far from understanding the mechanisms of clock entrainment by temperature cues.

\section{Additional genes: searching for functional roles}

ELF3 (EARLY FLOWERING 3) is an important component of the light signaling pathway to the clock. Initial studies showed that elf3 mutants displayed arrhythmic clock output expression in continuous light whereas rhythmicity was retained in constant darkness, suggesting a defect in the light input pathway to the clock (Hicks et al., 1996). In addition, the elf3 mutant plants did not discriminate different photoperiods, flowering as early in short days as in long days. In wild-type plants, $C A B$ induction is rhythmically repressed by the clock during the night, allowing a higher induction during the light period of the day (Millar and Kay, 1996). The circadian «gating» of $C A B$ acute induction is lost in elf3 mutants, leading to constitutive $C A B$ activation (McWatters et al., 2000). In a series of elegant studies, it was shown that the arrhythmicity in constant light of elf 3 mutants was due to a defect in the repression of light signals during the subjective night (McWatters et al., 2000). Both ELF3 transcript and protein rhythmically oscillate with a peak of expression at dusk, just when ELF3 is required to antagonize light signals to the clock (Covington et al., 2001, Hicks et al., 2001, Liu et al., 2001b, McWatters et al., 2000). Despite these significant advances, the exact mechanisms of ELF3 function in the light signaling pathway to the clock is not well understood. The elf3 mutants show more apparent phenotypes under red light conditions, indicating a connection with PHYB signaling. A physical interaction between PHYB and ELF3 has been described (Liu et al., 2001b). However, analysis of double mutant plants phyBelf3 showed an additive behavior, suggesting additional routes of ELF3 independently of PHYB (Reed et al., 2000).

Another gene with partially overlapping functions with $E L F 3$ is TIME FOR COFFEE (TIC, Hall et al., 2003). TiC mutant plants were shown to disrupt circadian rhyhtmicity of several clock outputs, arresting clock function in the subjective morning. Similar to ELF3 function at night, it was suggested a possible role for TIC in gating the clock during the day. Studies with double mutant ticlelf3 showed complete arrhythmia demonstrating that both genes are important in the functioning of the clock. The intimate connection between light signals and the circadian clock is also represented in SRR1 (sensitivity to red light reduced 1) function. Srr1 mutants were shown to be defective in PHYB-mediated signaling as well as in the normal expression of clock outputs (Staiger et al., 2003). The circadian phenotypes of srr1 mutants suggest that SRR1 activity might be required for normal oscillator function.

GIGANTEA ( $G /)$ is another gene that has been linked to the circadian clock. Gl encodes a novel, putative membrane protein that was initially identified by its highly delayed flowering phenotype under long days (Fowler et al., 1999). Additional experiments showed fluence-rate defects of the gi mutants in the circadian regulation of leaf movement and transcript oscillations, suggesting a connection of Gl with the light input to the clock (Fowler et al., 1999, Park et al., 1999). Rhythmic expression of $G$ / transcript was shown to be altered in gi mutants, suggesting that Gl could form a feedback loop required for normal clock function (Fowler et al., 1999, Park et al., 1999). In plants over-expressing CCA1 or LHY, the circadian expression of $G /$ was disrupted and reciprocally, the absence of Gl caused a reduction in the $C C A 1$ and $\angle H Y$ expression. The identification of interacting partners of GI might help to clarify GI function in this puzzled network. Recently, a report showed the interaction of SPINDLY (SPY), a negative regulator of gibberellin, with GI (Tseng et al., 2004). The report describes their involvement in light responses, flowering and rhythms in cotyledon movements. A further gi allele was identified in a screen for alterations in hypocotyl elongation under red light (Huq et al., 2000). These studies revealed that GI was also associated with PHYB function although the correlation of GI roles in photo-morphogenesis and in the light input to the clock remains to be elucidated.

\section{Transcriptional feedback loops}

Knowledge of circadian clock mechanisms in Arabidopsis thaliana has been aided by the formulation of circadian function in other circadian systems. The general mechanism of the clockwork seems to be conserved among organisms and it is based on negative feedback loops at the core of the oscillator (Dunlap, 1999, Young and Kay, 2001). This common theme, at its simplest, involves positive and negative components that mutually regulate their rhythmic abundance and/or activity. The oscillatory expression and regulation of clock components generates circadian rhythms that are translated to multiple clock outputs. While this mechanism has been firmly established in D. melanogaster, mammals and Neurospora crassa (Harmer et al., 2001), we are just beginning to ascertain clock mechanisms in higher plants (Figure 2). In Arabidopsis, the first working model for the plant clockwork came with the observation of a reciprocal regulation between CCA1/LHY and TOC1 (Alabadí et al., 2001). The two MYB proteins (LHY and CCA1) negatively regulated TOC1 expression that in turn positively induced $\angle H Y / C C A 1$. According to this model, the MYB transcription factors CCA1 and LHY might function as negative elements within this transcriptional loop, with a similar role to the one described for PERIOD (PER) and TIMELESS (TIM) in the circadian system of Drosophila, or FREQUENCY (FRQ) in the Neurospora clock. TOC1 might work as a positive component, similar to the clock components CYCLE (CYC) and CLOCK (CLK) in Drosophila, or WHITE COLLAR 1 and 2 (WC1 and WC2) in the circadian system of Neurospora. The repression of TOC1 might take place by the direct binding of CCA1/ LHY proteins to a region in the TOC1 promoter (denominated evening-element) (Alabadí et al., 2001, Harmer et al., 2000). Decreasing CCA1/LHY levels throughout the day causes a relieved in that repression, resulting in rising levels of TOC1 mRNA. The positive action of TOC1 on CCA1 and $L H Y$ expression thereby reinitiate the oscillatory cycle. The TOC1 activation of $C C A 1 / \angle H Y$ is probably indirect because at least three other components also positively participate in $C C A 1$ / 
LHY expression: ELF3 (Schaffer et al., 1998) GI (Fowler et al., 1999) and ELF4 (Doyle et al., 2002).

Additional levels of complexity are added to this regulatory loop since CCA1/ $\angle H Y$ as well as TOC1 are members of multigene families. Several homologues of CCA1/LHY (denominated REVEILLES) have been shown to circadianly oscillate with a peak of expression around dawn (Andersson et al., 1999). These additional MYB proteins could form homo- and hetero-interactions among them that could contribute to the oscillatory loop. TOC1 is also a member of a gene family composed of 4 more components (denominated pseudo-response regulators, $P R R 3,5,7$ and 9). Phenotypic analysis of mutant and over-expressing plants of individuals PRR has revealed alterations of period, phase and/or amplitude in some overt rhythms, changes in flowering time and affected sensitivity to red-light control of hypocotyl elongation (Mizuno, 2004). $P R R$ transcripts accumulate rhythmically in the order PRR9PRR7-PRR5-PRR3-TOC1 with peak levels after dawn from 2 $\mathrm{h}(P R R 9)$ to $10 \mathrm{~h}$ (TOC1, also known as $P R R 1)$ (Matsushika et al., 2000, Strayer et al., 2000). It is suggested that PRR participate in the light signaling within the circadian clock but are not required for rhythm generation (Mizuno, 2004).

Several studies have reported different circadian periods in various output genes suggesting regulation by multiple oscillators (Hall et al., 2002, Michael et al., 2003a, Thain et al., 2002). Based on their sensitivity to temperature, it was postulated the presence of two circadian clocks differentially controlling $C A B$ and CAT3 expression in Arabidopsis (Michael et al., 2003a). The partial overlapping expression of these genes opens up the possibility of the co-existence of at least two oscillators in the same cell although further experiments will be required to unequivocally confirm this hypothesis.

\section{Post-translational regulation of the plant clockwork}

Protein phosphorylation has been shown to be necessary in the functioning of the circadian clock in Drosophila, Neurospora and in humans. Various studies reveal that casein kinases (CK1 and CK2) are the main protein kinases involved in the phosphorylation of essential clock components (Dunlap, 2004, Edery, 1999, Loros and Dunlap, 2001, Toh et al., 2001). In Arabidopsis, the protein kinase CK2 was shown to phosphorylate in vitro the clock-associated protein CCA1 (Sugano et al., 1998). Overexpression of a CK2 regulatory subunit (denominated CKB3) affected the regulation of circadian rhythmicity, shortening the period of expression of several output genes (Sugano et al., 1999). By examining the effects of a constitutively-expressing CCA1 mutant that could not be phosphorylated by CK2, it was demonstrated that CCA1 phosphorylation by CK2 is important for the normal functioning of the Arabidopsis circadian clock (Daniel et al., 2004).

Detailed studies of LHY over-expressing plants showed that in constant light conditions, circadian rhythmicity was abolished although rhythmic expression was preserved under light-dark

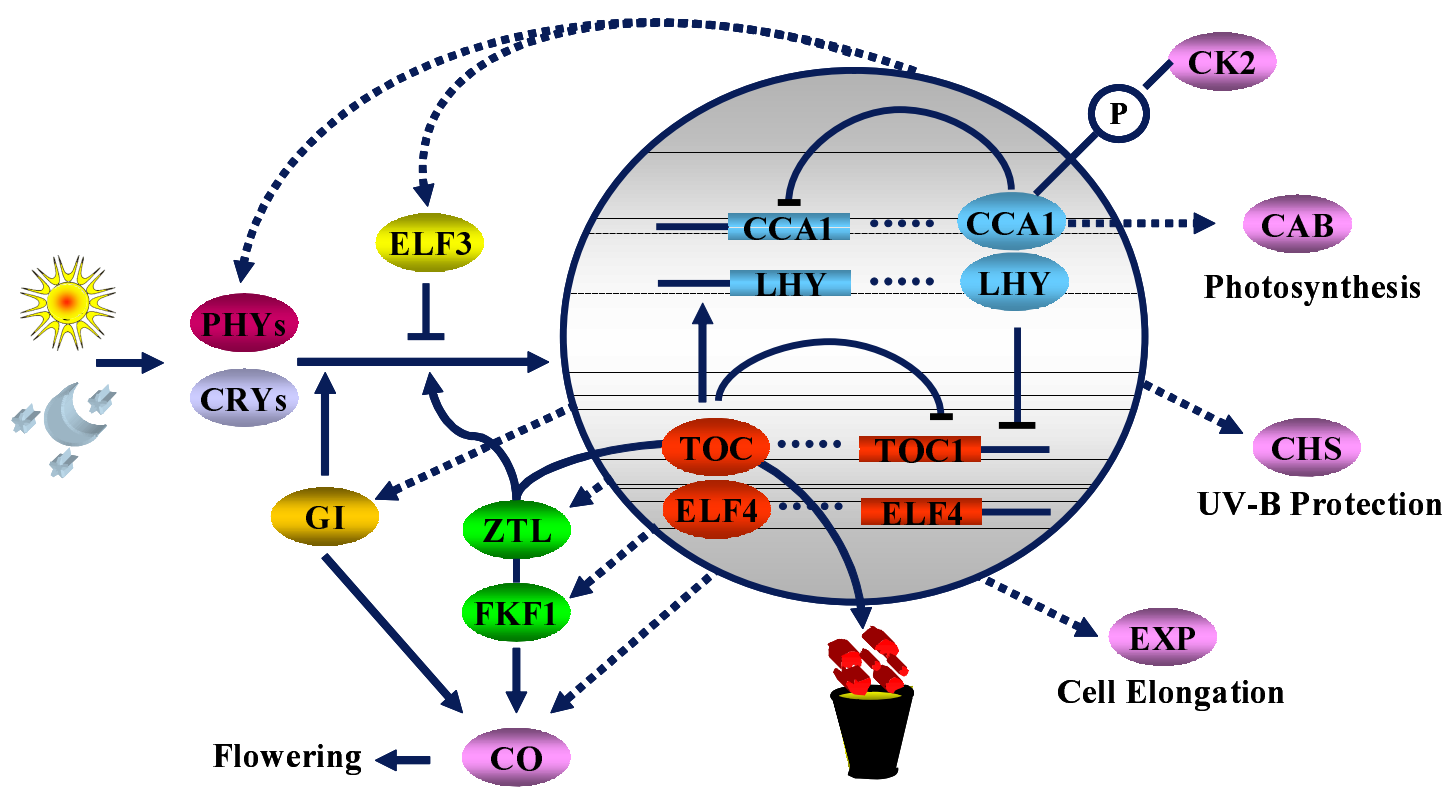

Fig. 2. Current molecular model of circadian clock signaling in the Arabidopsis. Light signals are perceived by a set of multiple photoreceptors, including phytochromes (PHYS) and cryptochromes (CRYS), that participate in the resetting of the clock. Light effects on the clock are also modulated by the action of both positive (GI) and negative light-signaling components (ELF3). At the core of the oscillator, a feedback loop generates rhythmicity by the reciprocal regulation between CCA1/LHY and TOC1/ELF4. CCA1 phosphorylation by CK2 is important for the normal functioning of the Arabidopsis circadian clock. The ZTL family (ZTL, FKF1 and LKP2) mediates between photoreceptors and the circadian clock. The targeted degradation of TOC1 protein by ZTL is essential in the control of circadian period by the clock. In the scheme, some of the genes and the physiological/metabolic processes regulated by the clock are represented: photosynthetic processes (CAB), lipid metabolism (CHS, CHALCONE SYNTHASE), cell elongation (EXP, EXPANSIN) and flowering-time regulation (CO). The transcription of $\mathrm{CO}$ is regulated by FKF1 and $\mathrm{Gl}$, whose transcription in turn is under the control of the circadian clock. The clock-controlled regulation of gene expression enables plants to anticipate and adapt to periodic changes in the environment. The gray shaded circle represents the feedback loop that is central for clock function in Arabidopsis; dotted arrows indicate gene expression regulated by the clock. 
cycles (Kim et al., 2003a). The rhythmic oscillations were correlated with light-induced regulation of $L H Y$ translation, causing high amplitude changes in LHY protein levels. The authors suggested that both translational induction and transcriptional repression of $L H Y$ at dawn contribute to the robustness and accuracy of the circadian oscillations.

Recent genetic and molecular evidence have also revealed that a precise post-translational regulation of the TOC1 protein is essential for clock function in Arabidopsis. The F-box protein ZEITLUPE (ZTL) was shown to be involved in the targeted degradation of TOC1 (Más et al., 2003b). In these studies, TOC1's physical interaction with ZTL was abolished by the $z t /-1$ mutation resulting in constitutive levels of TOC1 protein expression. The proteosome-mediated degradation of TOC1 protein occurs mainly during the dark period and requires a functional ZTL. It was demonstrated that the TOC1-ZTL interaction was important in the control of TOC1 protein stability and responsible for the accurate regulation of circadian period by the clock (Más et al., 2003b). The abundance of ZTL protein is also regulated by the proteosome. ZTL is more rapidly degraded at dawn when the protein reaches trough levels (Kim et al., 2003b). Post-translational regulation of protein stability through the proteosome pathway has been shown to be essential in clock function in the mammalian system and in Drosophila (Grima et al., 2002, Ko et al., 2002, Yagita etal., 2002). Together, these results suggest that tightly regulated protein degradation by the proteosome might be a conserved aspect in the regulation of the eukaryotic circadian clocks.

\section{Clock control of output gene expression}

Global analysis of clock-controlled gene expression is a useful tool for identifying new components of the circadian system and finding co-expressed genes integrated into common metabolic or

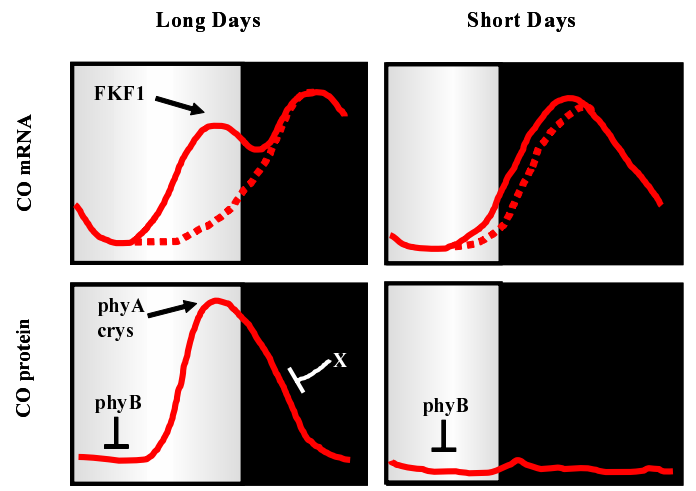

Fig. 3. Correlation of CO protein and mRNA expression with the photoperiodic control of Arabidopsis flowering time. CO mRNA levels are increased under long-days by the action of FKF1 protein that functions as a photoreceptor. Under long-days, the CO MRNA peak of expression at the end of the day is abolished in the fkf1 mutant plants (dotted line). The solid line indicates CO mRNA levels in wild-type plants. Blue and far-red light stabilize CO protein by the action of CRYS and PHYA. PHYB antagonizes the activity of CRYS and PHYA, especially in the morning. The combination of these regulatory activities results in robust floral promotion in long-days. physiologic pathways. The use of oligonucleotide microarrays has provided new insights into global transcription networks regulated by the Arabidopsis clock (Harmer et al., 2000, Schaffer et al., 2001). Clustering based on functional features of these genes has revealed how the clock enables plants to anticipate and adapt to the daily and seasonal fluctuations of light and temperature. A large set of photosynthetic genes were shown to be under clock control. All these genes exhibited a co-regulated expression, peaking around midday. Nine genes involved in energetically demanding processes such as nitrogen assimilation were expressed early in the day, when energy levels are increased by light harvesting. The expression of 23 genes encoding enzymes involved in the biosynthesis of photo-protective pigments was shown to peak coordinately before dawn. This pattern of expression might help to protect plants from the damaging effects of UV-B light. Another cluster of coordinately expressed genes was represented by a group involved in lipid modification which has been shown to oscillate and correlate with chilling tolerance. These genes have a peak of expression when most needed, just before dusk. These results illustrate the importance of the clock in compartmentalizing metabolic events to occur rhythmically at most advantageous time of the day (Harmer et al., 2000 , Schaffer et al., 2001). The current challenge is the followup of all these data to unravel the clock-controlled gene regulatory networks and their connection to physiology and development.

Microarray experiments combined with computational analysis have also helped to identify common motifs in the upstream regions of co-regulated genes (Harmer et al., 2000, Schaffer et al., 2001). A nine base-pair sequence, denominated EVENING ELEMENT ( $E E)$ was found to be over-represented in a cluster of genes, whose expression peaked at the end of the day. Mutational analysis confirmed the importance of the $E E$ in the oscillatory expression of COLD, CIRCADIAN RHYTHM 2 (CCR2) and in CATALASE 3 (CAT3) (Harmer et al., 2000, Michael and McClung, 2002). A region of the CCR2 promoter containing the $E E$ was shown to confer circadian rhythmicity to the $\beta$ GLUCORONIDASE reporter gene. The sequence of the EE (AAAATATCT) is identical to the one recognized in the TOC1 promoter by the transcriptional repressors CCA1 and LHY. The sequence is also highly similar to the one present in the $C A B$ promoter where CCA 1 binds acting as a positive regulator. These results suggest that CCA1 and LHY might control the expression of genes with opposite phases.

\section{Circadian regulation of plant growth}

The regulation of hypocotyl length is controlled by environmental signals (light and temperature) as well as by endogenous signaling pathways (e.g. giberellic acid, brassinosteroids, ethylene, abscisic acid, cytokinins). In constant light conditions, the pattern of hypocotyl growth displays rhythmic pauses near subjective dawn with a rapid elongation at subjective dusk. This rhythmicity is entrained by light-dark cycles and its period was shown to be shortened in the toc $1-1$ mutant, indicating that it is controlled by the circadian clock. TOC1 RNAi, toc1-2 mutants and mutants that over-express either CCA1 or LHY exhibit long hypocotyl phenotypes. Mutations in several other clock-associated components (such as ZTL, FKF1, ELF3, ELF4, GI, SRR1) also cause both circadian and hypocotyl-length phenotypes. The 
altered hypocotyl length in these clock mutants was shown to be due to severe circadian defects on the regulation of cell expansion (Dowson-Day and Millar, 1999). Interestingly, the correlation between the clock and hypocotyl length is not absolute; the toc11 mutant exhibits a short circadian period but does not have a hypocotyl phenotype. The fact that toc 1-1 mutant plants shorten multiple circadian outputs but still have wild-type hypocotyl length suggests that hypocotyl elongation during seedling de-etiolation could be controlled by different routes, dependent and independent of the clock. toc1-1 represents a mutation with affected clock function but with normal light-mediated de-etiolation response independent of the clock.

The role of the clock in the regulation of hypocotyl growth has been proposed to be based on the "gating" of the light signaling pathways in a similar way to the gated induction of CAB expression. When the gate is closed, the light signaling pathways are inhibited, even in the presence of light. This repression leads to the growth of hypocotyls. When the gate is open, the clock allows the light input signals, inhibiting hypocotyl elongation. As described above, ELF3 has an essential function in controlling this gating mechanism. Accordingly, loss-of-function elf3 mutant alleles result in a loss of rhythmic hypocotyl growth.

\section{Photoperiodic regulation of flowering time: a role for the circadian clock}

A crucial step of the plant life cycle is the transition from a vegetative stage to a reproductive mode (Mouradov et al., 2002, Searle and Coupland, 2004). Initiation of flowering occurs as a response to a number of environmental signals, including seasonal changes in day-length (Hayama and Coupland, 2004). The role of the circadian clock in the photoperiodism is illustrated by the fact that several mutants identified in Arabidopsis on the basis of their defective photoperiodic regulation of flowering also display altered circadian rhythms (e.g. ELF3, Hicks et al., 1996; LHY, Schaffer et al., 1998; Gl, Fowler et al., 1999, Park et al., 1999; ELF4, Doyle et al., 2002). Mutants originally isolated for their circadian defects, such as TOC1 (Millar etal., 1995, Somers etal., 1998b, Strayer et al., 2000) and ZTL (Somers et al., 2000), also showed a reduced sensitivity to day-length. Recently, an important progress has been made in the molecular understanding the circadian clock function in the photoperiodic control of flowering time. These studies set up the basis for a molecular understanding of the interactions between the circadian clock and the developmental control of flowering (Hayama and Coupland, 2004, Yanovsky and Kay, 2003). One of the key genes mediating the transition to flowering in Arabidopsis is CONSTANS (CO) (Putterill et al., 1995). Analysis of $C O$ expression reveals a rhythmic oscillation of both transcript and protein (Hayama and Coupland, 2003). An early flowering phenotype is observed in CO over-expressing plants although no circadian phenotypes are identified, indicating that $\mathrm{CO}$ is a clock output and mediates between the circadian system and initiation of flowering (Hayama and Coupland, 2003). The gene encodes a nuclear protein that contains a CCT motif (also found in TOC1 and in CO-like genes) and two B-box type zinc-finger domains, believed to mediate protein-protein interaction. Under long days, the peak of $\mathrm{CO}$ expression is at the end of the day and during the night. A different pattern is observed under short-day conditions with a majority of
CO expression during the night (Figure 3). The coincidence of higher levels of $C O$ with the light period has been proposed to be essential for the induction of flowering in long-days (SuárezLópez et al., 2001). The mechanism of CO action seems to be related with its involvement in FLOWERING LOCUS T (FT) activation. $F T$ is an essential gene triggering flowering in Arabidopsis and its expression has been tightly linked to the presence of active CO (Kardailsky et al., 1999, Kobayashi et al., 1999, Samach et al., 2000). The importance of $C O$ phase of expression has been demonstrated in the toc 1-1 mutant (with a short circadian period of $21 \mathrm{~h}$ ). $C O$ expression in this mutant is phase-shifted such that expression occurs during the light period in both long- and short-days. This pattern of $\mathrm{CO}$ expression results in a photoperiodic-insensitive early flowering. However, the photoperiodic control is restored when the toc 1-1 mutant plants are grown under light:dark cycles that match toc1-1 endogenous period of 21 hours (Yanovsky and Kay, 2002). These studies reveal the importance of the clock in controlling the phase of $C O$ expression. The coincidence of light with the $F T$ activation by $C O$ is a key event in the photoperiodic control of flowering time (Roden et al., 2002, Yanovsky and Kay, 2003) and two photoreceptors, PHYA and CRY2, have been shown to be involved in this process (Yanovsky and Kay, 2002).

Recent studies have also provided new insights into the mechanism that generates the diurnal pattern of $C O$ transcription. In the $f k f 1$ mutant, the high levels of $C O$ mRNA are strongly reduced and the daytime $\mathrm{CO}$ peak is abolished. Under long-days, FKF1 protein levels exhibit a diurnal pattern with a peak in the late day. However, under short-days the peak protein expression occurs during the early to mid nighttime, with low FKF1 protein levels during the day (Imaizumi et al., 2003). A model was proposed suggesting that under long-days, high levels of FKF1 protein and its direct activation by light occur simultaneously, generating the daytime peak of $C O$ mRNA.

Analysis of $\mathrm{CO}$ over-expressing plants revealed that $\mathrm{CO}$ protein accumulates under continuous white light, whereas $\mathrm{CO}$ levels are strongly reduced in constant darkness (Valverde et al., 2004). The dark-dependent instability of CO protein is due to an active degradation of $\mathrm{CO}$ protein by the proteosome. Furthermore, CO protein accumulates to high levels in continuous blue and far-red light, while the protein is rapidly degraded under constant red light. Under long-day conditions, CO protein shows a strong peak at the end of the day, whereas under short-days the protein displays a much weaker peak of expression at the early nighttime (Valverde et al., 2004). The regulation of CO protein abundance during the day seems to be mediated by PHYB, which decreases $\mathrm{CO}$ protein levels early in the morning. In contrast, PHYA and CRYs stabilize CO protein at the end of the day (Figure 3 ). These studies led to the conclusion that PHYB promotes $C O$ protein degradation and antagonizes the action of PHYA and CRYs in the morning (Hayama and Coupland, 2004).

Gene homologous to $C O$ and $F T$ have been identified in many species suggesting a conservation in the components of the Arabidopsis photoperiod pathway (Liu et al., 2001a, Yano et al., 2000, Kojima et al., 2002, Griffiths et al., 2003). Molecular and mechanistic studies in other plant species might help us to understand how the diversity in photoperiodic pathways was generated during evolution of different photoperiodic responses in plants. 


\section{Perspectives}

The precise control of gene expression by the circadian clock modulates the rhythmicity of physiological and developmental processes that are essential for plant survival. The clock is able to precisely recognize the time of the day in the light/dark cycle, allowing the anticipation of the environmental transitions. Elegant experiments performed in bacteria (Ouyang et al., 1998) and more recently in plants (Green et al., 2002, Michael et al., 2003b) have shown that this anticipation provides an adaptive advantage and increases the fitness of the organisms. The Arabidopsis circadian oscillator might be comprised by multiple interlocked feedback loops that generate and maintain circadian rhythmicity through a dynamic signaling network. Identifying clock components, their different levels of regulation and the interaction among them will be crucial in understanding mechanisms of action and influences of the clock on all circadianly-regulated processes. The first steps are already taken. New tools and experimental approaches will help to assemble the pieces into place and solve the intricate puzzle of the Arabidopsis circadian clock.

\section{Acknowledgments}

I thank T. Stratmann for critical reading of the manuscript. Our work on circadian rhythms is supported by grants (BIO2004-02144) from Spanish MEC and (FP6-012239) from EC.

\section{References}

ALABADÍ, D., OYAMA, T., YANOVSKY, M.J., HARMON, F.G., MÁS, P. and KAY, S.A. (2001). Reciprocal regulation between TOC1 and $L H Y / C C A 1$ within the Arabidopsis circadian clock. Science 293: 880-883.

ALABADÍ, D., YANOVSKY, M.J., MÁS, P., HARMER, S.L. and KAY, S.A. (2002). Critical role for CCA1 and LHY in maintaining circadian rhythmicity in Arabidopsis. Curr. Biol. 12: 757-761.

ANDERSSON, C.R., HARMER, S.L., SCHULTZ, T.F. and KAY, S.A. (1999). The REVEILLE (REV) family of DNA binding proteins and the circadian clock. In. Abstracts of the 10th international conference on Arabidopsis research, Melbourne, 4-8 July 1999. http://Arabidopsis.org/abstract_australia.pdf.

BARAK, S., TOBIN, E.M. andRONIS, C., SUGANO, S. and GREEN, R.M. (2000). All in good time: The Arabidopsis circadian clock. Trends Plant Sci. 5: 517-522.

COVINGTON, M.F., PANDA, S., LIU, X.L., STRAYER, C.A., WAGNER, D.R. and KAY, S.A. (2001). ELF3 modulates resetting of the circadian clock in Arabidopsis. Plant Cell 13: 1305-1315.

CRAIG, K.L. and TYERS, M. (1999). The F-box: A new motif for ubiquitin dependent proteolysis in cell cycle regulation and signal transduction. Prog. Biophys. Mol. Biol. 72: 299-328.

DANIEL, X., SUGANO, S. and TOBIN, E.M. (2004). CK2 phosphorylation of CCA1 is necessary for its circadian oscillator function in Arabidopsis. Proc. Natl. Acad. Sci. USA 101: 3292-7.

DEVLIN, P.F. and KAY, S.A. (2000). Cryptochromes are required for phytochrome signaling to the circadian clock but not for rhythmicity. Plant Cell 12: 2499-2510.

DEVLIN, P.F. and KAY, S.A. (2001). Circadian photoperception. Annu. Rev. Physiol. 63: 677-694.

DOWSON-DAY, M.J. and MILLAR, A.J. (1999). Circadian dysfunction causes aberrant hypocotyl elongation patterns in Arabidopsis. Plant J. 17:63-71.

DOYLE, M.R., DAVIS, S.J., BASTOW, R.M., MCWATTERS, H.G., KOZMA-BOGNAR, L., NAGY, F., MILLAR, A.J. and AMASINO, R.M. (2002). The ELF4 gene controls circadian rhythms and flowering time in Arabidopsis thaliana. Nature 419: 74-7.

DUNLAP, J.C. (1999). Molecular bases for circadian clocks. Cel/ 96: 271-290.

DUNLAP, J.C. (2004). Kinases and circadian clocks: Per goes it alone. Developmen- tal Cel/ 6: 160-161.

EDERY, I. (1999). Role of posttranscriptional regulation in circadian clocks: Lessons from Drosophila. Chronobiol. Int. 16: 377-414.

ERIKSSON, M.E. and MILLAR, A.J. (2003). The circadian clock. A plant's best friend in a spinning world. Plant Physiol 132: 732-8.

FOWLER, S., LEE, K., ONOUCHI, H., SAMACH, A., RICHARDSON, K., MORRIS, B., COUPLAND, G. and PUTTERILL, J. (1999). GIGANTEA : A circadian clockcontrolled gene that regulates photoperiodic flowering in Arabidopsis and encodes a protein with several possible membrane-spanning domains. EMBOJ.18: 4679-88.

GREEN, R.M., TINGAY, S., WANG, Z.Y. and TOBIN, E.M. (2002). Circadian rhythms confer a higher level of fitness to Arabidopsis plants. Plant Physiol. 129: 576-84.

GREEN, R.M. and TOBIN, E.M. (1999). Loss of the CIRCADIAN CLOCK-ASSOCIATED PROTEIN 1 in Arabidopsis results in altered clock-regulated gene expression. Proc. Natl. Acad. Sci. USA 96: 4176-9.

GRIFFITHS, S., DUNFORD, R.P., COUPLAND, G. and LAURIE, D.A. (2003). The evolution of CONSTANS-LIKE gene families in barley, rice and Arabidopsis. Plant Physiol. 131: 1855-1867

GRIMA, B., LAMOUROUX, A., CHELOT, E., PAPIN, C., LIMBOURG-BOUCHON, B. and ROUYER, F. (2002). The F-box protein slimb controls the levels of clock proteins period and timeless. Nature 420: 178-182.

HALL, A., BASTOW, R.M., DAVIS, S.J., HANANO, S., MCWATTERS, H.G., HIBBERD, V., DOYLE, M.R., SUNG, S., HALLIDAY, K.J., AMASINO, R.M. (2003). The TIME FOR COFFEE gene maintains the amplitude and timing of Arabidopsis circadian clocks. Plant Cell 15: 2719-2729.

HALL, A., KOZMA-BOGNAR, L., BASTOW, R.M., NAGY, F. and MILLAR, A.J. (2002) Distinct regulation of $C A B$ and $P H Y B$ gene expression by similar circadian clocks. Plant J. 32: 529-37.

HALLIDAY, K.J. and WHITELAM, G.C. (2003). Changes in photoperiod or temperature alter the functional relationships between phytochromes and reveal roles for PHYD and PHYE. Plant Physiol. 131: 1913-1920.

HARMER, S.L., HOGENESCH, J.B., STRAUME, M., CHANG, H., HAN, B., ZHU, T., WANG, X., KREPS, J.A. and KAY, S.A. (2000). Orchestrated transcription of key pathways in Arabidopsis by the circadian clock. Science 290: 2110-2113.

HARMER, S.L., PANDA, S. and KAY, S.A. (2001). Molecular bases of circadian rhythms. Annu. Rev. Cell Dev. Biol. 17: 215-253.

HAYAMA, R. and COUPLAND, G. (2003). Shedding light on the circadian clock and the photoperiodic control of flowering. Curr. Opin. Plant Biol. 6: 13-9.

HAYAMA, R. and COUPLAND, G. (2004). The molecular basis of diversity in the photoperiodic flowering responses of Arabidopsis and rice. Plant Physiol. 135: 677-84.

HICKS, K.A., ALBERTSON, T.M. and WAGNER, D.R. (2001). EARLY FLOWERING 3 encodes a novel protein that regulates circadian clock function and flowering in Arabidopsis. Plant Cell 13: 1281-1292.

HICKS, K.A., MILLAR, A.J., CARRÉ, I.A., SOMERS, D.E., STRAUME, M.D., MEEKSWAGNER, R. and KAY, S.A. (1996). Conditional circadian dysfunction of the Arabidopsis early-flowering 3 mutant. Science 274: 790-792.

HUQ, E., TEPPERMAN, J.M. and QUAIL, P.H. (2000). GIGANTEA is a nuclear protein involved in phytochrome signaling in Arabidopsis. Proc. Natl. Acad. Sci. USA 97: 9789-9794.

IMAIZUMI, T., TRAN, H.G., SWARTZ, T.E., BRIGGS, W.R. and KAY, S.A. (2003). FKF1 is essential for photoperiodic-specific light signalling in Arabidopsis. Nature 426: 302-6.

JARILLO, J.A., CAPEL, J., TANG, R.H., YANG, H.Q., ALONSO, J.M., ECKER, J.R and CASHMORE, A.R. (2001). An Arabidopsis circadian clock component interacts with both CRY1 and PHYB. Nature 410: 487-490.

JOHNSON, C.H. (2001). Endogenous timekeepers in photosynthetic organisms Annu Rev Physiol 63: 695-728.

KARDAILSKY, I., SHUKLA, V.K., AHN, J.H., DAGENAIS, N., CHRISTENSEN, S.K., NGUYEN, J.T., CHORY, J., HARRISON, M.J. and WEIGEL, D. (1999). Activation tagging of the floral inducer FT. Science 286: 1962-1965.

KIM, J.Y., SONG, H.R., TAYLOR, B.L. and CARRE, I.A. (2003a). Light-regulated translation mediates gated induction of the Arabidopsis clock protein LHY. EMBO J 22: 935-944.

KIM, W.-Y., GENG, R. and SOMERS, D.E. (2003b). Circadian phase-specific degra- 
dation of the F-box protein ZTL is mediated by the proteasome. Proc. Natl. Acad. Sci. USA 100: 4933-4938.

KO, H.W., JIANG, J. and EDERY, I. (2002). Role for SLIMB in the degradation of Drosophila PERIOD protein phosphorylated by DOUBLETIME. Nature 420.:673678.

KOBAYASHI, Y., KAYA, H., GOTO, K., IWABUCHI, M. and ARAKI, T. (1999). A pair of related genes with antagonistic roles in mediating flowering signals. Science 286: 1960-1962.

KOJIMA, S., TAKAHASHI, Y., KOBAYASHI, Y., MONNA, L., SASAKI, T., ARAKI, T. and YANO, M. (2002). Hd3a, a rice ortholog of the Arabidopsis FT gene, promotes transition to flowering downstream of $\mathrm{Hd} 1 \mathrm{under}$ short-day conditions. Plant Cell Physiol. 43: 1096-105.

LIU, J., YU, J., MCINTOSH, L., KENDE, H. and ZEEVAART, J.A. (2001a). Isolation of a CONSTANS ortholog from Pharbitis nil and its role in flowering. Plant Physiol 125: $1821-30$.

LIU, X.L., COVINGTON, M.F., FANKHAUSER, C., CHORY, J. and WAGNER, D.R. (2001b). ELF3 encodes a circadian clock-regulated nuclear protein that functions in an Arabidopsis PHYB signal transduction pathway. Plant Cel/ 13: 1293-1304.

LOROS, J.J. and DUNLAP, J.C. (2001). Genetic and molecular analysis of circadian rhythms in Neurospora. Annu. Rev. Physiol. 63: 757-794.

MÁS, P., ALABADI, D., YANOVSKY, M.J., OYAMA, T. and KAY, S.A. (2003a). Dual role of TOC1 in the control of circadian and photomorphogenic responses in Arabidopsis. Plant Cell 15: 223-236.

MÁS, P., DEVLIN, P.F., PANDA, S. and KAY, S.A. (2000). Functional interaction of PHYTOCHROME B and CRYPTOCHROME 2. Nature 408: 207-211.

MÁS, P., KIM, W.J., SOMERS, D.E. and KAY, S.A. (2003b). Targeted degradation of TOC 1 by ZTL modulates circadan function in Arabidopsis. Nature 426: 567-570.

MATSUSHIKA, A., MAKINO, S., KOJIMA, M. and MIZUNO, T. (2000). Circadian waves of expression of the APRR1/TOC 1 family of pseudo-response regulators in Arabidopsis thaliana: Insight into the plant circadian clock. Plant Cell Physiol. 41: 1002-1012.

MAZZELLA, M.A., BERTERO, D. and CASAL, J.J. (2000). Temperature-dependent internode elongation in vegetative plants of Arabidopsis thaliana lacking PHYTOCHROME B and CRYPTOCHROME 1. Planta 210: 497-501.

MCCLUNG, C.R. (2001). Circadian rhythms in plants. Annu. Rev. Plant Physiol. Plant Mol. Biol. 52: 139-162.

MCWATTERS, H.G., BASTOW, R.M., HALL, A. and MILLAR, A.J. (2000). The ELF3 zeitnehmer regulates light signaling to the circadian clock. Nature 408: 716-20.

MICHAEL, T.P. and MCCLUNG, C.R. (2002). Phase-specific circadian clock regulatory elements in Arabidopsis. Plant Physiol 130: 627-38.

MICHAEL, T.P., SALOME, P.A. and MCCLUNG, C.R. (2003a). Two Arabidopsis circadian oscillators can be distinguished by differential temperature sensitivity. Proc. Natt. Acad. Sci. USA 100: 6878-83.

MICHAEL, T.P., SALOME, P.A., YU, H.J., SPENCER, T.R., SHARP, E.L., MCPEEK, M.A., ALONSO, J.M., ECKER, J.R. and MCCLUNG, C.R. (2003b). Enhanced fitness conferred by naturally occurring variation in the circadian clock. Science 302: 1049-53.

MILLAR, A.J. (2004). Input signals to the plant circadian clock. J Exp Bot 55: 277-83.

MILLAR, A.J., CARRE, I.A., STRAYER, C.A., CHUA, N.H. and KAY, S.A. (1995). Circadian clock mutants in Arabidopsis identified by luciferase imaging. Science 267: 1161-3.

MILLAR, A.J. and KAY, S.A. (1996). Integration of circadian and phototransduction pathways in the network controlling cab gene transcription in Arabidopsis. Proc. Natl. Acad. Sci. USA 93: 15491-6.

MIZOGUCHI, T., WHEATLEY, K., HANZAWA, Y., WRIGHT, L., MIZOGUCHI, M., SONG, H.R., CARRE, I.A. and COUPLAND, G. (2002). $\angle H Y$ and CCA1 are partially redundant genes required to maintain circadian rhythms in Arabidopsis. Developmental Cell 2: 629-641.

MIZUNO, T. (2004). Plant response regulators implicated in signal transduction and circadian rhythm. Curr. Opin. Plant Biol. 7: 499-505.

MOURADOV, A., CREMER, F. and COUPLAND, G. (2002). Control of flowering time: Interacting pathways as a basis for diversity. Plant Cel/ 14 Suppl: S111-30.

NELSON, D.C., LASSWELL, J., ROGG, L.E., COHEN, M.A. and BARTEL, B. (2000). FKF1, a clock-controlled gene that regulates the transition to flowering in Arabidopsis. Cell 101: 331-340.
OUYANG, Y. and ERSSON, C.R., KONDO, T., GOLDEN, S.S. and JOHNSON, C.H. (1998). Resonating circadian clocks enhance fitness in cyanobacteria. Proc. Natl. Acad. Sci. USA 95: 8660-8664.

PARK, D.H., SOMERS, D.E., KIM, Y.S., CHOY, Y.H., LIM, H.K., SOH, M.S., KIM, H.J., KAY, S.A. and NAM, H.G. (1999). Control of circadian rhythms and photoperiodic flowering by the Arabidopsis gigantea gene. Science 285: 1579-1582.

PUTTERILL, J., ROBSON, F., LEE, K., SIMON, R. and COUPLAND, G. (1995). The CONSTANS gene of Arabidopsis promotes flowering and encodes a protein showing similarities to zinc finger transcription factors. Cel/ 80: 847-57.

REED, J.W., NAGPAL, P., BASTOW, R.M., SOLOMON, K.S., DOWSON-DAY, M.J., ELUMALAI, R.P. and MILLAR, A.J. (2000). Independent action of ELF3 and PHYB to control hypocotyl elongation and flowering time. Plant Physiol. 122: 1149-60.

RODEN, L.C., SONG, H.R., JACKSON, S., MORRIS, K. and CARRE, I.A. (2002). Floral responses to photoperiod are correlated with the timing of rhythmic expression relative to dawn and dusk in Arabidopsis. Proc. Natl. Acad. Sci. USA 99: 13313-8.

SAMACH, A., ONOUCHI, H., GOLD, S.E., DITTA, G.S., SCHWARZ-SOMMER, Z., YANOFSKY, M.F. and COUPLAND, G. (2000). Distinct roles of CONSTANS target genes in reproductive development of Arabidopsis. Science 288: 16131616.

SCHAFFER, R., LANDGRAF, J., ACCERBI, M., SIMON, V., LARSON, M. and WISMAN, E. (2001). Microarray analysis of diurnal and circadian-regulated genes in Arabidopsis. Plant Cell 13: 113-23.

SCHAFFER, R., RAMSAY, N., SAMACH, A., CORDEN, S., PUTTERILL, J., CARRÉ, I.A. and COUPLAND, G. (1998). The late elongated hypocotyl mutation of Arabidopsis disrupts circadian rhythms and the photoperiodic control of flowering. Cell 93: 1219-1229.

SCHULTZ, T.F., KIYOSUE, T., YANOVSKY, M., WADA, M. and KAY, S.A. (2001). A role for LKP2 in the circadian clock of Arabidopsis. Plant Cell 13: 2659-70.

SEARLE, I. and COUPLAND, G. (2004). Induction of flowering by seasonal changes in photoperiod. EMBO J.23: 1217-22.

SOMERS, D.E., DEVLIN, P.F. and KAY, S.A. (1998a). Phytochromes and cryptochromes in the entrainment of the Arabidopsis circadian clock. Science 282: $1488-1490$.

SOMERS, D.E., SCHULTZ, T.F., MILNAMOW, M. and KAY, S.A. (2000). ZE/TLUPE encodes a novel clock-associated PAS protein from Arabidopsis. Cel/ 101: 319329.

SOMERS, D.E., WEBB, A.A.R., PEARSON, M. and KAY, S.A. (1998b). The shortperiod mutant toc1-1, alters circadian clock regulation of multiple outputs throughout development in Arabidopsis thaliana. Development 125: 485-494.

STAIGER, D., ALLENBACH, L., SALATHIA, N., FIECHTER, V., DAVIS, S.J., MILLAR, A.J., CHORY, J. and FANKHAUSER, C. (2003). The Arabidopsis SRR1 gene mediates PHYB signaling and is required for normal circadian clock function. Genes Dev. 17: 256-268.

STRAYER, C.A., OYAMA, T., SCHULTZ, T.F., RAMAN, R., SOMERS, D.E., MÁS, P., PANDA, S., KREPS, J.A. and KAY, S.A. (2000). Cloning of the Arabidopsis clock gene TOC1, an autoregulatory response regulator homolog. Science 289: 768771.

SUÁREZ-LÓPEZ, P., WHEATLEY, K., ROBSON, F., ONOUCHI, H., VALVERDE, F. and COUPLAND, G. (2001). CONSTANS mediates between the circadian clock and the control of flowering in Arabidopsis. Nature 410: 1116-1120.

SUGANO, S. andRONIS, C., GREEN, R.M., WANG, Z.Y. and TOBIN, E.M. (1998). Protein kinase CK2 interacts with and phosphorylates the Arabidopsis circadian clock-associated 1 protein. Proc. Natl. Acad. Sci. USA 95: 11020-11025.

SUGANO, S. andRONIS, C., ONG, M.S., GREEN, R.M. and TOBIN, E.M. (1999). The protein kinase CK2 is involved in regulation of circadian rhythms in Arabidopsis. Proc. Natl. Acad. Sci. USA 96: 12362-12366.

THAIN, S.C., MURTAS, G., LYNN, J.R., MCGRATH, R.B. and MILLAR, A.J. (2002). The circadian clock that controls gene expression in Arabidopsis is tissue specific. Plant Physiol. 130: 102-10.

TOH, K.L., JONES, C.R., HE, Y., EIDE, E.J., HINZ, W.A., VIRSHUP, D.M., PTACEK, L.J. and FU, Y.-H. (2001). An hPER2 phosphorylation site mutation in familial advanced sleep phase syndrome. Science 291: 1040-1043.

TSENG, T.-S., SALOME, P.A., MCCLUNG, C.R. and OLSZEWSKI, N.E. (2004). SPINDLY and GIGANTEA interact and act in Arabidopsis thaliana pathways involved in light responses, flowering and rhythms in cotyledon movements. Plant 
Cell 16: 1550-1563.

VALVERDE, F., MOURADOV, A., SOPPE, W., RAVENSCROFT, D., SAMACH, A. and COUPLAND, G. (2004). Photoreceptor regulation of CONSTANS protein in photoperiodic flowering. Science 303: 1003-6.

WANG, Z.Y., KENIGSBUCH, D., SUN, L., HAREL, E., ONG, M.S. and TOBIN, E.M. (1997). A myb-related transcription factor is involved in the phytochrome regulation of an Arabidopsis LHCB gene. Plant Cell 9: 491-507.

WANG, Z.Y. and TOBIN, E.M. (1998). Constitutive expression of the circadian clock associated 1 (CCA1) gene disrupts circadian rhythms and suppresses its own expression. Cell 93: 1207-17.

YAGITA, K., TAMANINI, F., YASUDA, M., HOEIJMAKERS, J.H.J., VAN DER HORST, G.T.J. and OKAMURA, H. (2002). Nucleocytoplasmic shuttling and mCRY-dependent inhibition of ubiquitylation of the mPER2 clock protein.
EMBO J. 21: 1301-1314.

YANO, M., KATAYOSE, Y., ASHIKARI, M., YAMANOUCHI, U., MONNA, L., FUSE, T., BABA, T., YAMAMOTO, K., UMEHARA, Y., NAGAMURA, Y. (2000). Hd1, a major photoperiod sensitivity quantitative trait locus in rice, is closely related to the Arabidopsis flowering time gene CONSTANS. Plant Cell 12: 2473-2484.

YANOVSKY, M.J. and KAY, S.A. (2002). Molecular basis of seasonal time measurement in Arabidopsis. Nature 419: 308-12.

YANOVSKY, M.J. and KAY, S.A. (2003). Living by the calendar: How plants know when to flower. Nat. Rev. Mol. Cell Biol. 4: 265-275.

YANOVSKY, M.J., MAZZELLA, M.A. and CASAL, J.J. (2000). A quadruple photoreceptor mutant still keeps track of time. Curr. Biol 10: 1013-1015.

YOUNG, M.W. and KAY, S.A. (2001). Time zones: A comparative genetics of circadian clocks. Nat. Rev. Gen. 2: 702-715. 